\title{
EL GÉNERO IMPATIENTINUM MORDVILKO, 1914 (HEMIPTERA, APHIDIDAE, MACROSIPHINI) EN AMÉRICA, CON LA DESCRIPCIÓN DE TRES NUEVAS ESPECIES
}

\author{
J. M. Nieto Nafría ${ }^{1}$, R. Peña-Martínez ${ }^{2}$, \\ M. P. Mier Durante ${ }^{1} \&$ N. Pérez Hidalgo ${ }^{1}$
}

\section{RESUMEN}

J. M. Nieto Nafría, R. Peña-Martínez, M. P. Mier Durante \& N. Pérez Hidalgo. 2012. El género Impatientinum Mordvilko, 1914 (Hemiptera, Aphididae, Macrosiphini) en América, con la descripción de tres nuevas especies. Graellsia, 68(2): 313-324.

El estudio de los ejemplares del género Impatientinum Mordvilko, 1914 de la collection générale d'aphides del Muséum national d'Historie naturelle de París (Francia) y de la Colección de Aphididae de México en la Universidad Nacional Autónoma de México ha permitido establecer 3 especies: I. chiriquense $\mathbf{n}$. sp. (con la descripción de hembras ápteras y aladas) de Panamá, I. holmani n. sp. (con la descripción de hembras ápteras, aladas y de machos) e I. oaxacense n. sp. (con la descripción de ápteras) de México. Se describen también las vivíparas aladas y se mejora el conocimiento de las vivíparas ápteras de $I$. americanum. Se discute la ubicación genérica de las especies del género presentes en América. Se facilita la clave para la identificación de las hembras vivíparas ápteras de las especies americanas del género, que puede sustituir a la clave de Blackman \& Eastop para las especies de pulgones que viven sobre plantas del género Cuphea P.Browne (Myrtales, Lythraceae) en el mundo.

Palabras clave: América; Aphididae; Macrosiphini; pulgones; áfidos; Impatientinum; especies nuevas.

\section{ABSTRACT}

J. M. Nieto Nafría, R. Peña-Martínez, M. P. Mier Durante \& N. Pérez Hidalgo. 2012. The genus Impatientinum Mordvilko, 1914 (Hemiptera, Aphididae, Macrosiphini) from America, with the description of three new species. Grael/sia, 68(2): 313-324 (in Spanish).

The study of the specimens of genus Impatientinum Mordvilko, 1914 from the collection générale d'aphides del Muséum national d'Historie naturelle, Paris (France) and the Colección de Aphididae de México at the Universidad Nacional Autónoma de México has allowed to establish 3 species: I. chiriquense $\mathbf{n}$. sp. (with the description of the apterous and alate females) from Panama, I. holmani n. sp. (with the description of the apterous and alate females and males) and $I$. oaxacense $\mathbf{n}$. sp. (with the description of the apterae) from México. The viviparous alate females of $I$. americanum are described and knowledge of the apterous 
females of this species is improved. The generic position of the American species of Impatientinum is discussed. An identification key for the apterous females of the American species of Impatientinum is presented; this key can substitute the key by Blackman and Eastop for the World's aphids on Cuphea P.Browne (Myrtales, Lythraceae).

Key words: America; Aphididae; Macrosiphini; aphids; Impatientinum; new species.

\section{Introducción}

El género Impatientinum Mordvilko, 1914 está clasificado en la tribu Macrosiphini Wilson 1910 (1887) (Hemiptera, Aphididae, Aphidinae), se divide en 2 subgéneros (el nominotípico y Neoimpatientinum Agarwala, Mondal \& Raychaudhuri, 1982) y encuadra 6 especies. Dos de ellas son americanas: $I$. (I.) americanum Remaudière, 1981 de México (estado de Hidalgo) e $I$. (I.) paranaense de Carvalho, Cardoso \& Lazzari, 2004 del Brasil (estado de Paraná); las otras tres especies del subgénero nominotípico son paleárticas, y la única especie del subgénero Neoimpatientinum es oriental (de Carvalho et al., 2004; Blackman \& Eastop, 2006, 2011; Nieto Nafría et al., 2011; Favret, 2012).

Con el fin de conocer mejor la diversidad del género Impatientinum en América se han estudiado todos los ejemplares de ese género de la collection générale d'aphides del Muséum national d'Historie naturelle, París (Francia) [a partir de aquí: M.n.H.n.] y de la colección de Aphididae de México en la Universidad Nacional Autónoma de México, Facultad de Estudios Superiores Iztacala [a partir de aquí: F.E.S.I.].

\section{Material y métodos}

Los ejemplares estudiados ha sido: A) el holotipo y los paratipos de I. americanum, vivíparas ápteras, M.n.H.n.; B) paratipos de I. paranaense, vivíparas ápteras y aladas, colección de la Universidade Federal do Paraná, Curitiba, Paraná, Brasil; C) vivíparas ápteras y aladas de varias procedencias de I. asiaticum Nevsky, 1929, I. balsamines (Kaltenbach, 1862) e I. impatiens (Shinji, 1922), M.n.H.n.; D) vivíparas ápteras y aladas y machos de Impatientinum spp., M.n.H.n. y F.E.S.I., como se detalla en los apartados dedicados a $I$. americanum y a cada una de las nuevas especies. Algunos ejemplares de ambas colecciones tuvieron que remontarse debido al mal estado de conservación de las preparaciones.
Se han tenido en cuenta las aportaciones de Remaudière (1981), Heie (1994), de Carvalho et al. (2004) y Blackman \& Eastop (2006). Las medidas se han tomado siguiendo los procedimientos habituales (Nieto Nafría \& Mier Durante, 1998). Los dibujos se han realizado con una cámara clara incorporada al microscopio y las fotografías se han tomado con una cámara digital Leica DC incorporada también al microscopio y han sido procesadas con el programa IM 1000, versión 1.10.

\section{Resultados y discusión}

Impatientinum americanum Remaudière, 1981

MATERIAL EXAMINADO: Los ejemplares tipo, y también 27 especímenes con los siguientes datos de captura: MéXICO: Oaxaca, El Estudiante (1970 m), Cuphea sp., 19-VIII-1981, R. Peña \& J. Holman leg., 11 vivíparas ápteras y 1 alada, M.n.H.n. (registro G. Remaudière 012389), 4 ápteras, F.E.S.I. (registro R. Peña 99); MÉxico: México, Cerro Tláloc (2700 m aprox.), Cuphea sp., 5-VIII-1981, R. Peña \& J. Holman leg., 3 ápteras, M.n.H.n. (registro G. Remaudière 012391), 6 ápteras y 2 aladas, F.E.S.I. (registro R. Peña 0553).

HEMBRAS VIVÍPARAS ÁPTERAS (Figs. 1A, 2A, 3A; Tabla 1): Caracteres cualitativos como en la descripción original (Remaudière, 1981) salvo en los cornículos la intensidad de la reticulación, que en varios ejemplares es más evidente que en los tipos, y el reborde apical, que no siempre está tan marcado como en los tipos.

HEMBRAS VIVÍPARAS ALADAS (Figs. 1F, 2F, 3F; Tabla 2): Antenas casi homogéneamente pigmentadas, con una minúscula zona proximal del artejo III pálida. Sensorios secundarios presentes solamente en el artejo III, casi alineados y algo más abundantes en la mitad proximal que en la distal. Fémures pálidos en su parte proximal y tan pigmentados como los tarsos en su 1/4-1/3 distal. Venas alares pigmentadas pero no rebordeadas. Dorso del abdomen sin placa discal; segmentos 2 a 7 con escleritos marginales menos pigmentados que los intersegmentarios, y parcialmente fusionados los de cada lado en los segmentos 5-7; segmento 8 con pequeños escleritos 

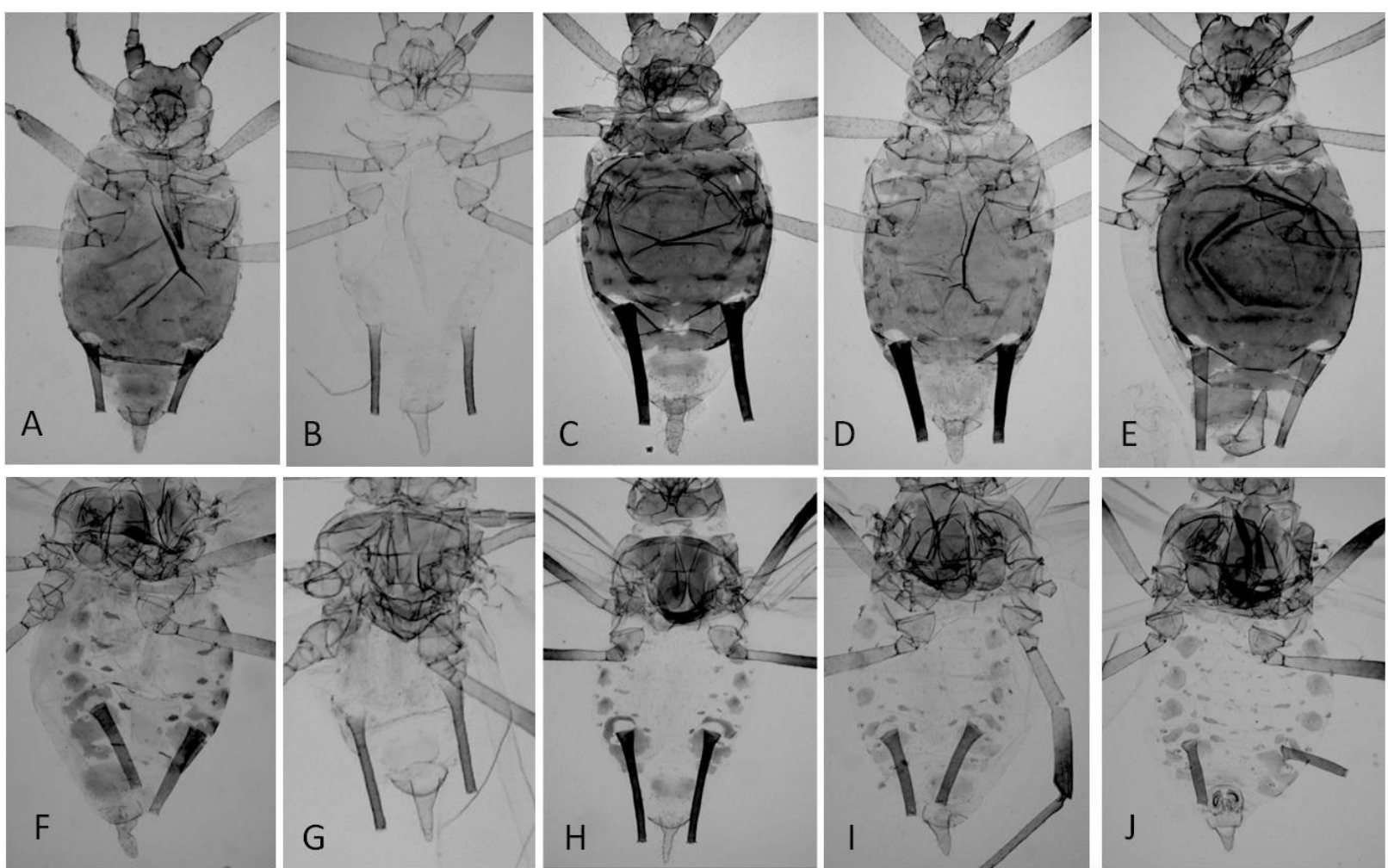

Fig. 1.- Impatientinum americanum (A, F), I. paranaense (B, G), I. chiriquense sp. nov. (C, H), I. holmani sp. nov. (D, I, J), I. oaxacense sp. nov. $(E)$; hembra vivípara áptera $(A, B, C, D, E)$, hembra vivípara alada $(F, G, H, I)$, macho $(J)$.

Fig. 1.- Impatientinum americanum (A, F), I. paranaense $(B, G)$, l. chiriquense sp. nov. $(C, H)$, I. holmani sp. nov. (D, I, J), I. oaxacense sp. nov. (E); apterous viviparous female $(A, B, C, D, E)$, alate viviparous female $(F, G, H, I)$, male $(J)$.

setíferos. Reticulación cornicular algo más extensa y mejor definida que en las ápteras, a las que se asemejan en el resto de caracteres cualitativos.

BIOLOGÍA, DISTRIBUCIÓN: Los nuevos datos mantienen la relación exclusiva de la especie con el género Cuphea y amplían hacia el sur su área de distribución.

Discusión: La identificación de las 3 aladas descritas se ha basado fundamentalmente en el número de setas complementarias del artejo apical del rostro, que coincide con el de las ápteras de I. americanum recogidas al mismo tiempo. Las diferencias en la pigmentación de antenas y de patas y en la reticulación cornicular que se aprecian entre ellas, son semejantes a las que se presentan entre las ápteras y aladas de $I$. paranaense, I. chiriquense sp. n. e $I$. holmani sp. n. (de Carvalho et al., 2004, y este mismo artículo).
Impatientinum chiriquense Nieto Nafría \& Mier Durante n. sp.

MATERIAL TIPO: Holotipo: hembra vivípara áptera (número 2 de la serie de medidas), PANAMÁ: Chiriquí. Volcán (1300 m aprox.), Cuphea infundibulum, 7-IV-1987, G. Remaudière leg., M.n.H.n. (registro G. Remaudière núm. 010703). Paratipos: 5 hembras vivíparas ápteras y 3 hembras vivíparas aladas con los mismos datos que el holotipo.

HEMBRAS VIVÍPARAS ÁPTERAS (Figs. 1C, 2C, 3C; Tabla 1): En vida posiblemente tengan color negro brillante por el dorso y verde por el vientre, con antenas, patas y cornículos pardo oscuros o negros. En preparación destaca el color pardo de la extensa placa discal, de las patas y sobre todo de antenas y cornículos. Cabeza sin espículas y con algunas estrías muy finas y pequeñas en dorso y vientre. Seno frontal poco profundo de bordes divergentes, y tubérculo mediano de altura semejante a 0,5 veces la profundidad del seno. Antenas intensa- 
Tabla 1.- Caracteres cuantitativos de las hembras vivíparas ápteras de l. americanum (material tipo y nuevos datos), I. holmani sp. nov., I. oaxacense n. sp. e l. chiriquense n. sp.- Los límites mínimo y máximo en $I$. americanum se encuentran habitualmente entre los "nuevos datos", los límites que corresponden a la serie tipo se marcan con negrita.

Table 1.- Quantitative features of the apterous viviparous females of $I$. americanum (typical series and new data), $I$. holmani sp. nov., $I$. oaxacense n. sp. e $I$. chiriquense n. sp.- The maximal and minimal limits of the features ranges of $I$. americanum are usually within the new data, when they correspond to the typical series they are in bold characters.

\begin{tabular}{|c|c|c|c|c|c|c|c|c|c|}
\hline \multirow{3}{*}{ VIVÍPARAS ÁPTERAS } & \multicolumn{4}{|c|}{ I. americanum } & \multicolumn{2}{|c|}{ I. holmani } & \multirow[t]{3}{*}{ I. oaxacense } & \multicolumn{2}{|c|}{ I. chiriquense } \\
\hline & \multicolumn{2}{|c|}{ serie tipo } & \multicolumn{2}{|c|}{ nuevos datos } & \multirow[b]{2}{*}{ MíN. } & \multirow[b]{2}{*}{ MÁX. } & & \multirow[b]{2}{*}{ MíN. } & \multirow[b]{2}{*}{ MÁX. } \\
\hline & MíN. & MÁX. & MíN. & MÁX. & & & & & \\
\hline cuerpo [mm] & 1,690 & 2,200 & 1,450 & 2,600 & 1,675 & 2,325 & 2,275 & 1,680 & 1,970 \\
\hline antena $[\mathrm{mm}]$ & 2,080 & 2,370 & 1,765 & 2,610 & 1,370 & 2,425 & & 1,910 & 2,320 \\
\hline antena / cuerpo [veces] & 1,08 & 1,23 & 0,954 & 1,256 & 0,699 & 1,170 & & 1,100 & 1,300 \\
\hline artejo antenal III [mm] & 0,46 & 0,57 & 0,39 & 0,65 & 0,43 & 0,52 & 0,70 & 0,46 & 0,64 \\
\hline artejo antenal IV [mm] & 0,31 & 0,40 & 0,26 & 0,46 & 0,23 & 0,46 & 0,54 & 0,30 & 0,55 \\
\hline artejo antenal V [mm] & 0,29 & 0,39 & 0,26 & 0,47 & 0,29 & 0,41 & 0,47 & 0,27 & 0,45 \\
\hline artejo antenal $\mathrm{VI}$, base [mm] & 0,13 & 0,15 & 0,12 & 0,17 & 0,12 & 0,15 & 0,15 & 0,12 & 0,16 \\
\hline artejo antenal VI filamento terminal $[\mathrm{mm}]$ & 0,64 & 0,72 & 0,53 & 0,73 & 0,60 & 0,93 & & 0,53 & 0,66 \\
\hline $\begin{array}{l}\text { artejo antenal VI filamento terminal / } \\
\text { artejo antenal III [veces] }\end{array}$ & 1,2 & 1,4 & 0,91 & 1,63 & 1,20 & 1,98 & & 1,00 & 1,20 \\
\hline $\begin{array}{l}\text { artejo antenal VI: filamento terminal / } \\
\text { base [veces] }\end{array}$ & 4.5 & 5.0 & 4.08 & 5,31 & 4,29 & 6,89 & & 4,50 & 5,30 \\
\hline $\begin{array}{l}\text { setas artejo antenal III [ } \mu \mathrm{m}] \\
\text { setas artejo antenal III / diámetro }\end{array}$ & & & 12,5 & 25,0 & 20,0 & 27,5 & 15,0 & 22,0 & 27,0 \\
\hline basal artejo antenal III [veces] & 0,3 & 0,5 & 0,5 & 0,8 & 0,7 & 1,0 & 0,4 & 0,8 & 1,0 \\
\hline $\begin{array}{l}\text { sensorios secundarios artejo antenal III } \\
\text { [cantidad] }\end{array}$ & 1 & 3 & (0) 1 & 5 & 0 & 3 & 4 & 2 & 5 \\
\hline setas vértex $[\mu \mathrm{m}]$ & & & 25,0 & 42,5 & 27,5 & 42,5 & 27,5 & 27,0 & 37,0 \\
\hline $\begin{array}{l}\text { setas vértex / diámetro basal artejo } \\
\text { antenal III [veces] }\end{array}$ & & & 0,9 & 1,5 & 0,9 & 1,4 & 0,7 & 1,0 & 1,4 \\
\hline artejo apical del rostro [mm] & 0,14 & 0,15 & 0,12 & 0,16 & 0,15 & 0,17 & 0,145 & 0,144 & 0,166 \\
\hline $\begin{array}{l}\text { artejo apical del rostro: longitud / } \\
\text { anchura en la base [veces] }\end{array}$ & 2,6 & 3,2 & 2,3 & 3,2 & 2,7 & 3,8 & 2,6 & 2,6 & 3,2 \\
\hline setas artejo apical rostro [cantidad] & 14 & 16 & 10 & $16(18)$ & $(17) 18$ & 22 & 7 & 19 & 33 \\
\hline $\begin{array}{l}\text { setas tibias posteriores }[\mu \mathrm{m}] \\
\text { setas tibias posteriores / diámetro }\end{array}$ & & & 20,0 & 35,0 & 20,0 & 37,5 & 17,5 & 29,0 & 40,0 \\
\hline basal artejo antenal III [veces] & $<1$ & $<1$ & 0,6 & 1,1 & 0,7 & 1,3 & 0,5 & 1,1 & 1,3 \\
\hline $\begin{array}{l}2 .^{\circ} \text { artejo tarsos posteriores }[\mathrm{mm}] \\
\text { artejo apical rostro } / 2 .^{\circ} \text { artejo tarsos }\end{array}$ & 0,10 & 0,12 & 0,08 & 0,11 & 0,09 & 0,12 & 0,11 & 0,10 & 0,11 \\
\hline $\begin{array}{l}\text { posteriores [veces] } \\
\text { setas espinales segmento }\end{array}$ & 1,20 & 1,35 & 1,32 & 1,63 & 1,30 & 1,90 & 1,32 & 1,4 & 1,6 \\
\hline abdominal $3[\mu \mathrm{m}]$ & 20,0 & 20,0 & 7,5 & 20,0 & 10,0 & 20,0 & 20,0 & & \\
\hline $\begin{array}{l}\text { setas espinales segmento abdominal } 3 \text { / } \\
\text { diámetro basal artejo antenal III [veces] }\end{array}$ & 0,5 & 0,5 & 0,3 & 0,6 & 0,4 & 0,7 & 0,5 & & \\
\hline cornículo $[\mathrm{mm}]$ & 0,320 & 0,450 & 0,270 & 0,620 & 0,395 & 0,595 & 0,475 & 0,370 & 0,520 \\
\hline cuerpo / cornículo [veces] & 4,500 & 5,500 & 4,185 & 5,480 & 3,649 & 4,722 & 4,789 & & \\
\hline $\begin{array}{l}\text { cornículo / anchura cabeza [veces] } \\
\text { cornículo: longitud / anchura }\end{array}$ & 0,80 & 1,05 & 0,72 & 1,13 & 0,96 & 1,23 & 0,97 & & \\
\hline en la base [veces] & & & 3,75 & 6,06 & 3,91 & 6,29 & 5,00 & & \\
\hline $\begin{array}{l}\text { cornículo: longitud / anchura en } \\
\text { el medio [veces] } \\
\text { cornículo: porción reticulada / }\end{array}$ & 9,00 & 12,00 & 6,08 & 10,80 & 6,92 & 11,10 & 9,05 & & \\
\hline cornículo [veces] & & & 0,06 & 0,13 & 0,06 & 0,12 & 0,29 & & \\
\hline $\begin{array}{l}\text { cornículo: líneas en la reticulación } \\
\text { cornicular [cantidad] } \\
\text { cornículo, anchuras: cornicular: de la porción }\end{array}$ & 2 & 3 & 2 & 5 & 3 & 5 & 8 & 2 & 4 \\
\hline reticulada /en el medio [veces] & 1,40 & 1,50 & 0,85 & 1,38 & 0,88 & 1,33 & 1,05 & & \\
\hline
\end{tabular}


Tabla 1. Cont.

\begin{tabular}{|c|c|c|c|c|c|c|c|c|c|}
\hline \multirow{3}{*}{ VIVÍPARAS ÁPTERAS } & \multicolumn{4}{|c|}{ I. americanum } & \multicolumn{2}{|c|}{ I. holmani } & \multirow[t]{3}{*}{ I. oaxacense } & \multicolumn{2}{|c|}{ I. chiriquense } \\
\hline & \multicolumn{2}{|c|}{ serie tipo } & \multicolumn{2}{|c|}{ nuevos datos } & \multirow[b]{2}{*}{ MíN. } & \multirow[b]{2}{*}{ MÁX. } & & \multirow[b]{2}{*}{ MíN. } & \multirow[b]{2}{*}{ MÁX. } \\
\hline & MíN. & MÁX. & MíN. & MÁX. & & & & & \\
\hline \multicolumn{10}{|l|}{ cornículo, anchuras: del reborde / } \\
\hline de la porción reticulada [veces] & & & 1,10 & 1,56 & 1,00 & 1,50 & 1,22 & & \\
\hline cornículo / cola [veces] & 1,50 & 2,10 & 1,52 & 2,07 & 1,87 & 2,33 & 1,79 & 1,60 & 2,00 \\
\hline setas segmento abdominal 7 [cantidad] & 6 & 6 & 4 & 7 & 4 & 7 & 6 & 5 & 6 \\
\hline setas segmento abdominal 8 [cantidad] & 6 & 6 & 4 & 7 & 4 & 6 & 6 & & \\
\hline setas segmento abdominal $8[\mu \mathrm{m}]$ & & & 22,5 & 62,5 & 20,0 & 45,0 & 32,5 & & \\
\hline \multicolumn{9}{|l|}{ setas segmento abdominal / diámetro } & \\
\hline cola $[\mathrm{mm}]$ & 0,20 & 0,24 & 0,18 & 0,29 & 0,18 & 0,27 & 0,27 & 0,22 & 0,25 \\
\hline cola: longitud / anchura en la base [veces] & 1,30 & 1,50 & 1,40 & 2,30 & 1,45 & 2,26 & 1,66 & 1,95 & 2,68 \\
\hline setas caudales [cantidad] & 7 & 7 & 6 & 7 & 5 & 9 & 7 & 7 & 9 \\
\hline $\begin{array}{l}\text { setas posteriores de la placa genital } \\
\text { [cantidad] }\end{array}$ & 12 & 16 & 8 & 14 & 10 & 16 & 10 & 11 & 13 \\
\hline
\end{tabular}

mente pigmentadas en toda su extensión; artejos I y II con algunas espínulas y estrías, y con setas puntiagudas y robustas (éstas contrastan con las del flagelo antenal que son cortas y romas); artejo III liso salvo una pequeña porción basal y con sensorios secundarios próximos a su base; artejo IV con imbricaciones débiles y esparcidas; artejos V y VI con imbricaciones muy evidentes. Rostro tan pigmentado como la cabeza; segundo artejo levemente espinuloso; artejo apical triangular alargado y con algunas espínulas. Tórax con amplias bandas segmentarias dorsales, algo rugosas y sin espínulas, y con escleritos intersegmentarios poco o nada evidentes. Protórax sin papilas marginales. Setas torácicas más cortas que las dorsales de la cabeza. Coxas amarillo parduscas así como los trocánteres y la mayor parte de fémures y tibias; tarsos, porción distal de las tibias y 1/5-1/4 distal de los fémures oscuros, al menos tanto como la placa discal. Setas femorales y tibiales respectivamente más cortas que el diámetro de la sutura trocántero-femoral y que el diámetro del artejo al nivel de su inserción. Primer segmento de los tarsos con 4 o 5 setas, excepcionalmente 3 en alguna pata. Abdomen con una placa discal amplia, que incluye a los escleritos estigmáticos y a los intersegmentarios y que deja libre un anillo en torno a la inserción de los cornículos, bien pigmentada y casi completamente lisa (la zona del segmento 6 lleva alguna espínula). Sin papilas marginales. Setas ventrales más largas que las dorsales y apuntadas. Cornículos más oscuros que la placa discal y que los segmentos antenales I y II; largos, de borde exterior ligeramente convexo y borde interior sinuoso, presentando su mayor anchura en la base y en el tercer cuarto de su longitud; reborde apical de anchura variable pero normalmente muy evidente; casi lisos en el dorso de 1/3-1/2 proximal, reticulados en el ápice y con espínulas o escamas gruesas en el resto. Segmento abdominal 7 con una banda esclerotizada dorsal amplia (incluye los escleritos estigmáticos) y con espínulas dispersas. Dorso del segmento $8 \mathrm{mem}$ branoso, con algunos grupos de espínulas formando escamas, raramente con escleritos setíferos muy poco marcados y con setas apuntadas o romas, y siempre más largas que las de los segmentos anteriores. Placa genital con espínulas pequeñas y formando grupos. Cola lanceolada, relativamente corta y muy pálida, como la placa anal; setas caudales relativamente finas y apuntadas.

HEMBRAS VIVÍPARAS ALADAS (Figs. 1H, 2H, 3H; Tabla 2): Semejantes en general a las vivíparas ápteras, pero con los fémures más intensamente pigmentados en el $1 / 2-3 / 5$ distal, sin placa discal abdominal, con escleritos marginales en los segmentos abdominales 2-6 menos pigmentados que los intersegmentarios y escleritos setíferos en el segmento 7; con espínulas en los escleritos marginales y zonas de los segmentos 6 a 8 . Sensorios secundarios del artejo III casi alineados y extendidos sobre casi toda su longitud. Venas alares pigmentadas pero no rebordeadas. Caracteres cuantitativos en la tabla 2.

BIOLOGÍA, DISTRIBUCIÓN: La especie vive sobre Cuphea inphundibulum y quizás sobre otras espe- 
Tabla 2.- Caracteres cuantitativos de hembras vivíparas aladas de I. americanum (primeros datos), I. holmani n. sp. e I. chiriquense n. sp. y de machos de I. holmani n. sp.

Table 2.- Quantitative features of the alatae viviparous females of $I$. americanum (first data), $I$. holmani n. sp. and $I$. chiriquense $\mathbf{n}$. sp. and males of I. holmani $\mathbf{n} . \mathbf{s p}$.

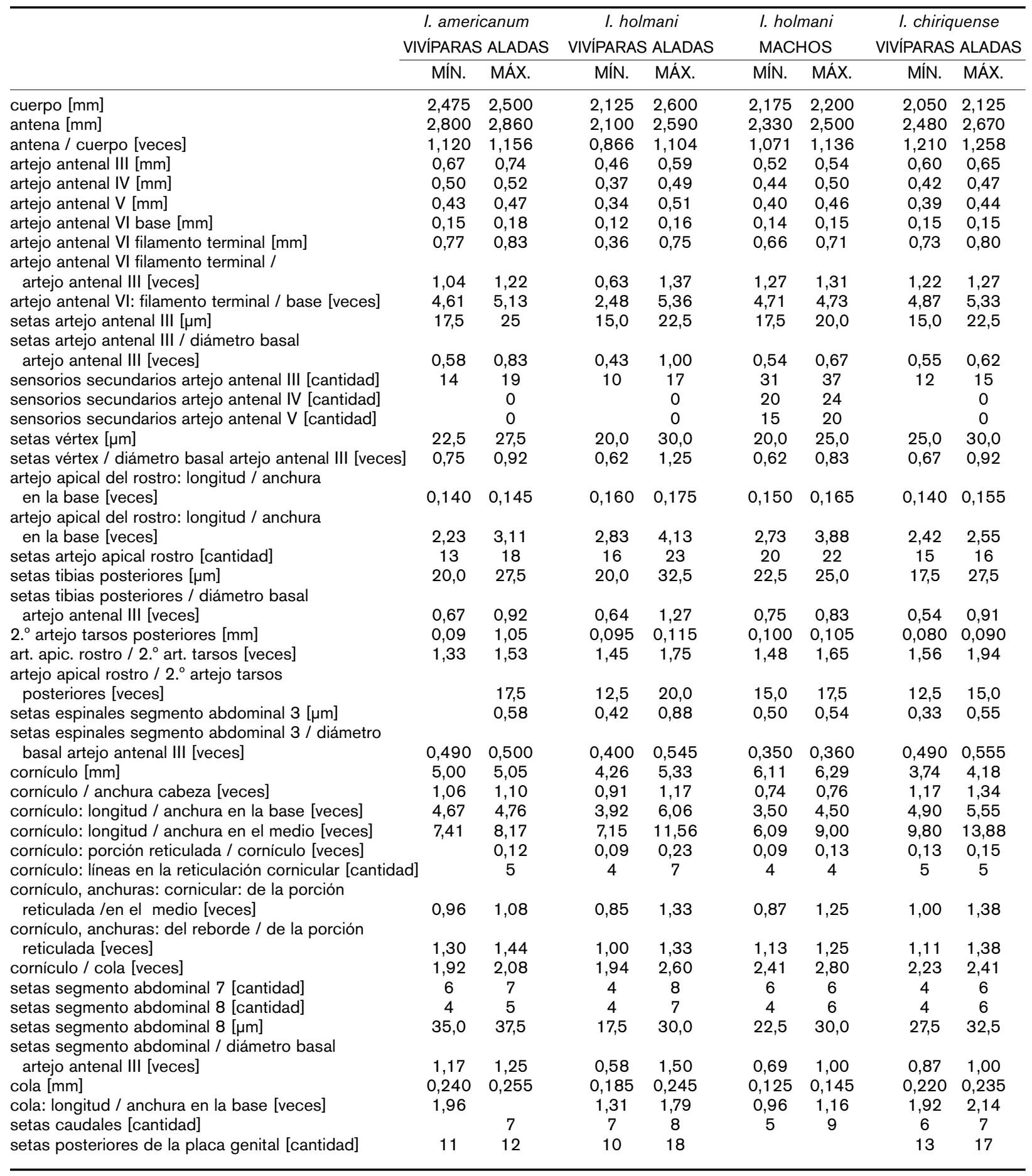



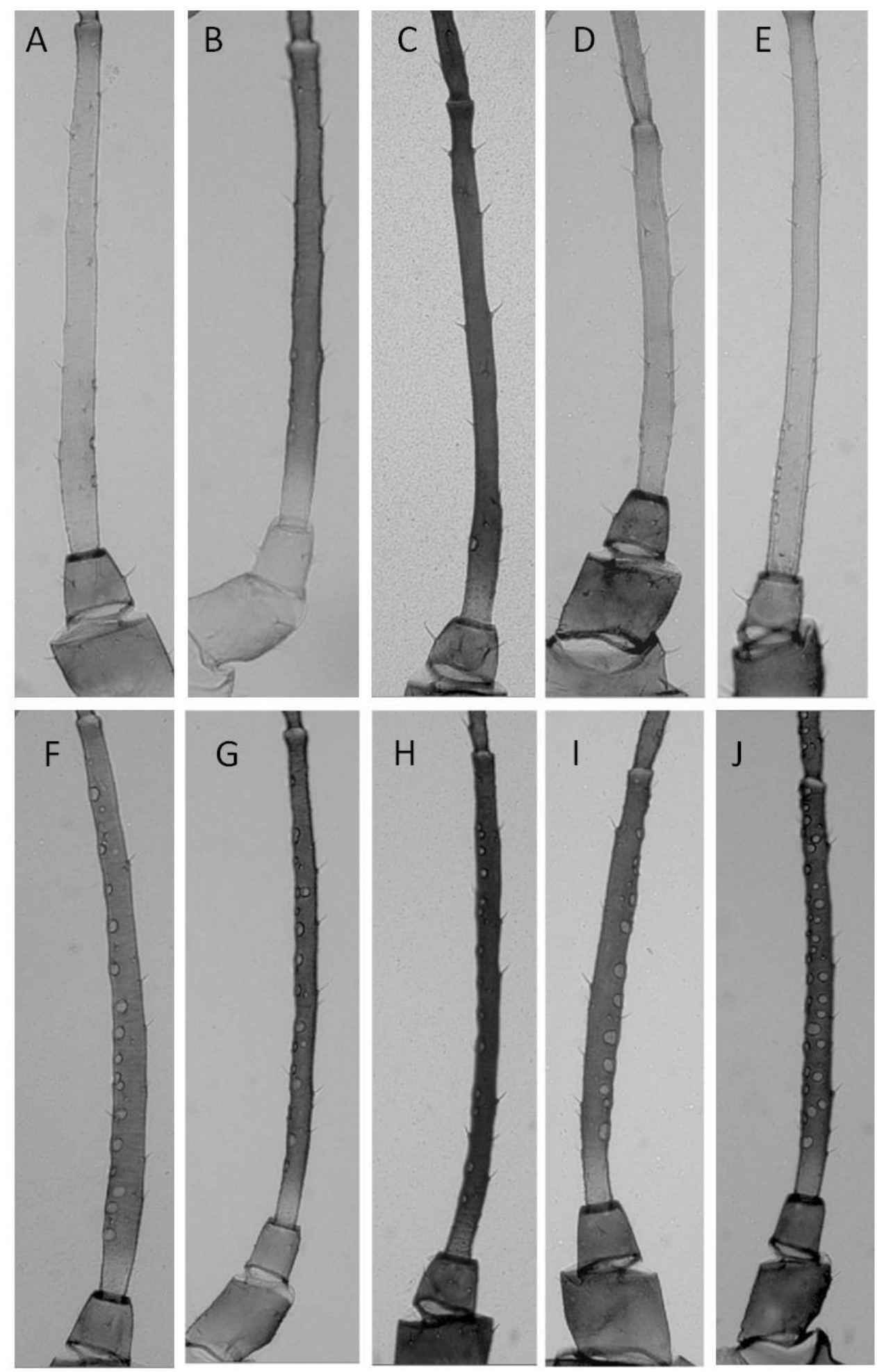

Fig. 2.- Antena (en parte) de Impatientinum americanum (A, F), l. paranaense (B, G), l. chiriquense sp. nov. (C, H), I. holmani sp. nov. $(D, I, J)$, I. oaxacense sp. nov. (E); hembra vivipara áptera $(A, B, C, D, E)$, hembra vivipara alada $(F, G, H, I)$, macho $(J)$.

Fig. 2.- Antenna (in part) of Impatientinum americanum (A, F), l. paranaense (B, G), l. chiriquense sp. nov. (C, H), I. holmani sp. nov. $(D, I, J)$, I. oaxacense sp. nov. (E); apterous viviparous female $(A, B, C, D, E)$, alate viviparous female $(F, G, H, I)$, male $(J)$. 
cies de Cuphea. Su área de distribución puede estar limitada a la cordillera de Talamanca o extenderse por otras áreas montañosas de América Central.

ETIMOLOGíA: El nombre específico chiriquense es un adjetivo neutro, para concordar con Impatientinum que es neutro (Cortés Gabaudan et al., 2011), en nominativo singular, construido con el nombre de la provincia panameña de Chiriquí y el sufijo ense, según recomienda el Código Internacional de Nomenclatura Zoológica para estos casos.

DISCUSIÓN: Las ápteras de Impatientinum chiriquense se asemejan a las de $I$. paranaense (Figs. 1B, 2B, 3B): por la extensa e intensa pigmentación de las antenas, carácter que diferencia a ambas especies de $I$. americanum y de las dos nuevas especies que se establecen a continuación. I. chiriquense e I. paranaense se pueden diferenciar rápidamente entre sí porque: $\left.1^{\circ}{ }^{\circ}\right)$ los artejos antenales I y II son más pálidos que los restantes en I. paranaense y tan oscuros como los restantes en I. chiriquense; $2 .^{\circ}$ ) la pigmentación de las placas dorsales es poco intensa en I. paranaense pero es intensa en I. chiriquense; $3 .^{\circ}$ ) hay diferencias de importancia en la longitud absoluta del artejo apical del rostro, la proporción entre esa longitud y la del segundo de los tarsos posteriores y la cantidad de sus setas accesorias (ver tablas 1 y 2). Las aladas de ambas especies (Figs. 1G, 1H, 2G, 2H, 3G, 3H) se pueden separar por los criterios $2 .^{\circ}$ y $3 .^{\circ}$ de separación de las ápteras.

\section{Impatientinum holmani Peña-Martínez \& Nieto Nafría} n. sp.

MATERIAL TIPO: Holotipo: hembra vivípara áptera (número 6 de la serie de medidas, en la misma preparación microscópica que la número 7), MÉXICO: Michoacán, Lagunillas, Puerto el Tigre, (2420 m), Cuphea sp., 20-X-1981, R. Peña \& J. Holman leg. (registro de captura 276), M.n.H.n. (registro G. Remaudière núm. 012388). Paratipos: 18 vivíparas ápteras, 29 vivíparas aladas y 4 machos con los mismos datos que el holotipo; 1 vivípara áptera, MÉXICO: Michoacán, Lagunillas, Puerto el Tigre, (2420 m), Cuphea sp., 22-X-1981 (R. Peña \& J. Holman leg. (registro de captura 286e); 5 hembras vivíparas ápteras, México: Michoacán, Estación Ajuno, 21-X-1981, Cuphea sp., R. Peña \& J. Holman leg. (registro de captura 277d); M.n.H.n., F.E.S.I. y colección de la Universidad de León.

HEMBRAS VIVÍPARAS ÁPTERAS (Figs. 1D, 2D, 3D; Tabla 1): En vida posiblemente de color negro o pardo oscuro brillante por el dorso y verde por el vientre, con cornículos pardo oscuros y con antenas y patas pardas. En preparación destaca el color pardo de la mayor parte del dorso del cuerpo y de parte de las patas y antenas. Cabeza sin espículas y con algunas pequeñas estrías en dorso y vientre. Seno frontal poco profundo de bordes divergentes, y con un tubérculo mediano elevado 0,5 veces la profundidad del seno aproximadamente. Artejos antenales I, II y VI aproximadamente tan pigmentados como la cabeza, extremos distales de los artejos III-V algo pigmentados, resto de la antena pálido. Artejo I con algunas espínulas gruesas, sobre todo en el vientre y en una franja externa del dorso; artejo II con pocas espínulas, dispersas y pequeñas; ambos con setas robustas y mucho más largas que las del flagelo antenal; artejo III liso salvo una pequeña porción basal, más extensa en el vientre que en el dorso, que lleva espínulas formando escamas; artejos IV-VI imbricados. Sensorios secundarios pequeños limitados al 1/5 proximal del artejo III. Setas de los artejos antenales III a VI cortas y romas, semejantes a las setas del dorso del tórax y de los segmentos abdominales 1-7, y a la mayoría de las setas de los apéndices, pero más cortas que las del dorso de la cabeza. Rostro tan pigmentado como la cabeza; segundo artejo algo espinuloso; artejo apical esbelto y liso. Tórax con amplias bandas esclerotizadas dorsales, segmentarias, sin espínulas, las del protórax y mesotórax algo rugosas, y con escleritos intersegmentarios poco marcados; la banda metatorácica está fusionada a veces con la abdominal discal. Protórax con papilas marginales muy pequeñas. Patas pálidas en general, con tarsos, porción distal de las tibias y 1/4-1/3 distal de los fémures parduscos. Setas femorales menores que el diámetro de la sutura trocántero-femoral, y setas tibiales habitualmente más cortas que el diámetro de la tibia al nivel de su inserción. Primer artejo de los tarsos con 4 o 5 setas, sin que predomine una u otra cantidad en las patas de uno u otro par. Abdomen con placa discal amplia, que incluye a los escleritos intersegmentarios y a los estigmáticos y deja libre un anillo en torno a la inserción de los cornículos, parda y casi completamente lisa (la zona correspondiente al segmento 6 lleva alguna espínula). Normalmente con papilas marginales ( 3 o más) en los segmentos abdominales 2-4(5), protuberantes aunque normalmente menores que el alveolo de las setas marginales. Setas ventrales más largas que las dorsales y apuntadas. Cornículos más oscuros que la placa discal, largos, moderadamente robustos, con su 
borde exterior ligeramente convexo y su borde interior sinuoso, reticulados en el ápice, casi lisos en 1/3-1/2 proximal, con espínulas o escamas gruesas en el resto de su longitud, y con reborde apical pequeño a inconspicuo. Segmento 7 con una banda esclerotizada dorsal amplia, que incluye los escleritos estigmáticos, lleva espínulas dispersas y a veces está fusionada completamente o en parte con la placa discal. Dorso del segmento 8 membranoso o raramente con escleritos setíferos muy poco marcados, con algunos grupos de espínulas formando escamas, setas romas y más largas que las de segmentos anteriores y en algunos ejemplares con $1 \mathrm{o}$ 2 papilas espinales minúsculas. Placa genital con espínulas pequeñas y dispersas. Cola lanceolada y relativamente corta, pálida como la placa anal, con setas largas finas y apuntadas.

HEMBRAS VIVÍPARAS ALADAS (Figs. 1I, 2I, 3I; Tabla 2): Seme-jantes en general a las vivíparas ápteras, pero sin placa discal abdominal, con escleritos marginales en los segmentos 2-7, menos pigmentados que los intersegmentarios y a veces con escleritos setíferos en el segmento 7 y más raramente en el 8; con espínulas dorso-abdominales menos densas y menos marcadas; antenas intensamente pigmentadas, con una mínima porción proximal del artejo III pálida; y sensorios secundarios casi alineados y extendidos sobre la totalidad del artejo III; fémures más extensa (1/3-2/5 distal) e intensamente pigmentados. Venas de las alas anteriores bien pigmentadas pero no rebordeadas.

Machos (Figs. 1J, 2J, 3J; Tabla 2): Son alados. Semejantes a las vivíparas aladas, aunque de menor tamaño y con la cola más corta y sensorios secundarios en los artejos antenales III, IV y V.

BIOLOGÍA, DISTRIBUCIÓN: La especie es holocíclica, como demuestra la presencia de machos, y está relacionada con plantas del género Cuphea, aunque no es posible indicar con cuál o cuales especies. Su área de distribución incluye zonas del estado de Michoacán y quizás de otras entidades territoriales del centro de México.

ETimología: El nombre específico holmani es un sustantivo en genitivo singular, construido según dispone el Código Internacional de Nomenclatura Zoológica a partir del apellido de Jaroslav Holman, eminente afidólogo checo, que fue uno de los recolectores de los ejemplares estudiados, como lo ha sido de otros muchos pulgones conservados en la colección de Aphididae de

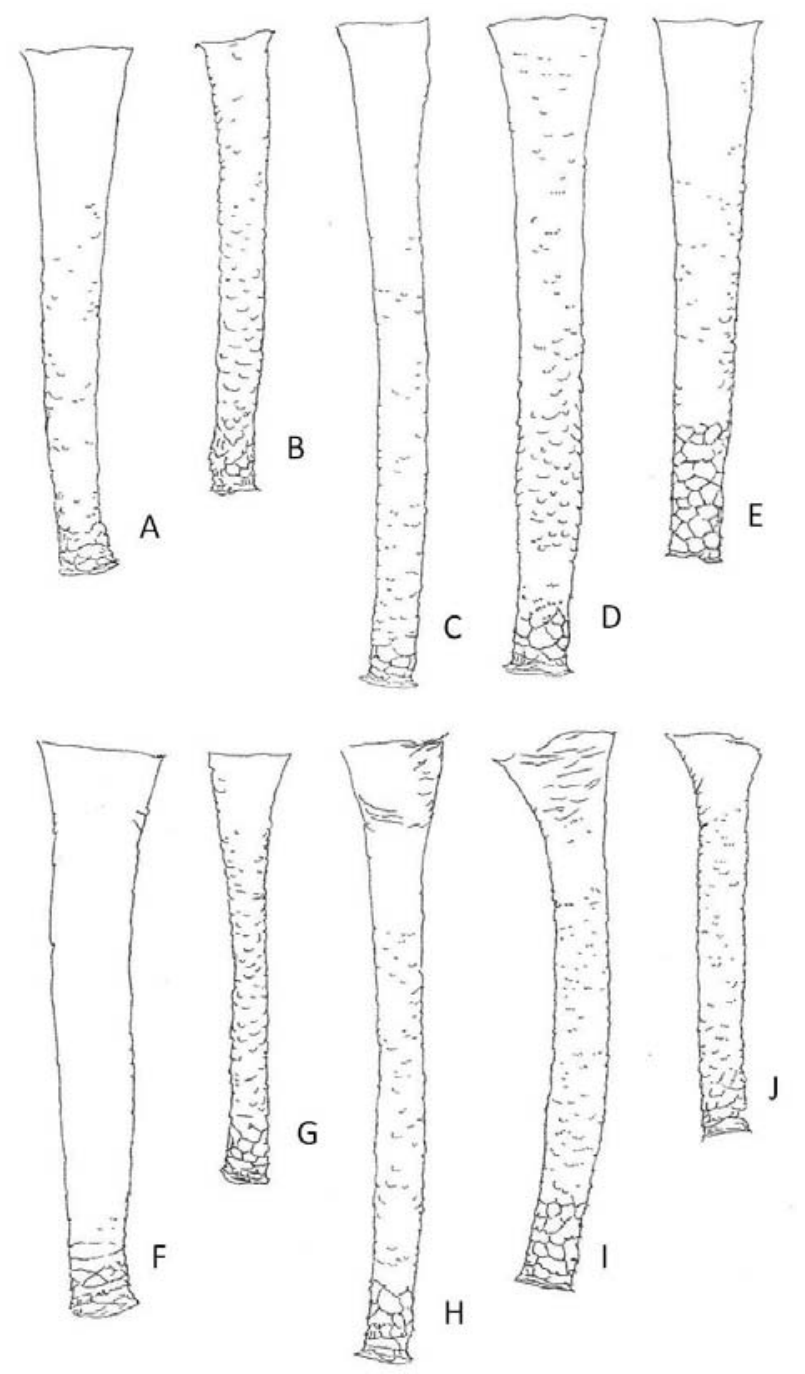

Fig. 3.- Cornículo (sin pigmentación) de Impatientinum americanum $(\mathrm{A}, \mathrm{F})$, I. paranaense $(\mathrm{B}, \mathrm{G})$, l. chiriquense sp. nov. $(C, H)$, I. holmani sp. nov. $(D, I, J)$, l. oaxacense sp. nov. $(E)$; hembra vivípara áptera $(A, B, C, D, E)$, hembra vivípara alada $(F, G, H, I)$, macho $(J)$.

Fig. 3.- Siphunculus (without pigmentation) of Impatientinum americanum (A, F), I. paranaense $(\mathrm{B}, \mathrm{G})$, I. chiriquense sp. nov. $(C, H)$, I. holmani sp. nov. (D, I, J), I. oaxacense sp. nov. (E); apterous viviparous female (A, B, $C, D, E)$, alate viviparous female $(F, G, H, I)$, male $(J)$.

México de la Facultad de Estudios Superiores Iztacala de la Universidad Nacional Autónoma de México.

DisCUSIÓN: Las hembras vivíparas ápteras de $I$. holmani, junto con las de I. americanum, se sepa- 
ran de las de I. paranaense e I. chiriquense por las antenas pálidas en general, con los artejos I y II algo pigmentados. Las de I. holmani se separan de las de I. americanum por: $\left.1 .^{\circ}\right)$ la pigmentación de los fémures, pálidos en americanum, oscuros en una porción dorsal y apical en holmani, $2 .^{\circ}$ ) las setas accesorias del artejo apical del rostro, en holmani 18 a 22 (excepcionalmente 17), y en americanum 14 a 16 (excepcionalmente 18 ), $3 .^{\circ}$ ) las setas en el primer artejo de los tarsos, en americanum 3 o raramente 4 , y en holmani 4 o $5,4 .^{\circ}$ ) el seno frontal, menos profundo en americanum, y $5^{\circ}$ ) la ornamentación antenal, evidente aunque tenue en holmani.

\section{Impatientinum oaxacense Nieto Nafría \& Pérez} Hidalgo n. sp.

MATERIAL TIPO: Holotipo: hembra vivípara áptera (compartiendo preparación microscópica con una hembra vivípara áptera de Impatientinum americanum), MÉxICO: Oaxaca, El Estudiante (1970 m), Cuphea sp., 19-VIII-1981, R. Peña \& J. Holman leg., M.n.H.n. (registro G. Remaudière núm. 012389b).

HEMBRAS VIVÍPARAS ÁPTERAS (Figs. 1E, 2E, 3E; Tabla 1): En vida posiblemente tengan color negro brillante por el dorso y verde por el vientre, y cornículos pardos y antenas y patas amarillentas. En preparación presentan color pardo por la extensa, intensa y homogénea pigmentación del dorso del cuerpo. Cabeza sin espículas y con pequeñas estrías tanto dorsales como ventrales. Seno frontal profundo (tanto como la distancia entre las setas de los tubérculos laterales) de bordes divergentes, y con un pequeño tubérculo mediano. Antenas pálidas en general, pero con los artejos antenales I, II y VI tan pigmentados como la cabeza y los extremos distales de los artejos III a V algo pigmentados. Artejo I con algunas espínulas gruesas, sobre todo en su vientre y en una franja externa del dorso; artejo II con alguna espínula gruesa en el vientre; ambos con setas robustas y más largas que las del flagelo antenal. Artejo III liso en general, con espínulas formando escamas en una pequeña porción proximal y ventral; resto del flagelo antenal imbricado. Setas de los artejos antenales cortas y romas, semejantes a las setas del dorso del tórax y de los segmentos abdominales 1-7, y a la mayoría de las setas de los apéndices. Sensorios secundarios pequeños y más o menos alineados en el cuarto basal del artejo III. Setas frontales y del vértex mucho más largas que las del artejo antenal III. Rostro tan pigmenta- do como la cabeza; segundo artejo muy espinuloso; artejo apical esbelto y liso. Tórax con amplias bandas dorsales esclerotizadas y escleritos intersegmentarios bien marcados; la banda mesotorácica lleva espínulas y rugosidades en toda su superficie y la protorácica solamente en las zonas marginales. Sin papilas marginales en el protórax. Patas apenas pigmentadas, poco más que el vientre del abdomen; tarsos y una pequeña porción distal de las tibias casi tan oscuros como la placa discal abdominal y fémures con una mancha subapical dorsal muy tenue. Setas femorales y tibiales respectivamente más cortas que el diámetro de la sutura trocántero-femoral y que el diámetro del segmento al nivel de su inserción. Primer artejo de los tarsos con 3 setas. Abdomen con una placa discal amplia que no alcanza los escleritos estigmáticos, en la que resaltan los escleritos intersegmentarios por estar más pigmentados, con un anillo membranoso en torno a la inserción de los cornículos, y con algunas espínulas en la zona correspondiente al segmento 6 . Sin papilas marginales o espinales en el abdomen. Setas ventrales apuntadas y más largas que las dorsales. Cornículos tan pigmentados como la placa discal, casi cilíndricos, robustos, con reborde bien marcado, casi lisos en su 1/4 proximal, nítidamente reticulados en su $1 / 4$ distal y con espínulas o escamas evidentes en el resto de su longitud. Segmento 7 con una banda dorsal esclerotizada amplia (incluyendo los estigmas) y espinulosa. Segmento 8 con escleritos setíferos, escamoso, y con setas más largas que las de segmentos anteriores y apuntadas. Placa genital cubierta de espínulas. Cola lanceolada y poco pigmentada, como la placa anal; setas caudales fuertes y apuntadas.

Biología, Distribución: Los datos disponibles de I. oaxacense sp. n. son muy escasos (ver apartado de tipos) y solamente permiten informar que la especie se desarrolla sobre plantas del género Cuphea, y que su área de distribución incluye al estado de Oaxaca.

ETIMOLOGÍA: El nombre específico, oaxacense, es un supuesto adjetivo latino referido a Oaxaca, en nominativo singular neutro porque Impatientinum es neutro (Cortés Gabaudan et al., 2011).

DISCUSIÓN: Impatientinum oaxacense se diferencia de las otras especies americanas del género porque en ella la reticulación de los cornículos es mucho más extensa y está más marcada, y porque el artejo apical del rostro lleva pocas setas accesorias (7). 


\section{El género Impatientinum en América}

Remaudière (1981) no expresó ninguna duda en la adscripción genérica de Impatientinum americanum, a pesar de la distribución (paleártica) y plantas hospedadoras (Balsamiaceae) de las especies adscritas al género en ese momento; también debió tener en cuenta los caracteres de Catamergus Oestlund, 1922, que es un género neártico con dos especies, una de ellas monoica sobre Impatiens. Las hembras vivíparas ápteras de ambos géneros se diferencian por la esclerotización dorsal, con amplia placa discal en Impatientinum y sin ella en Catamergus, y además porque (Foottit \& Richards 1993): a) los cornículos están reticulados en el ápice, pero en Catamergus están mucho más ornamentados y son relativamente más estrechos y cortos; b) las setas dorsales son cortas, pero en Catamergus son aguzadas; y c) suele haber papilas marginales en el protórax y en gran parte de los segmentos abdominales 2 a 6 y puede haber espinales en el segmento 8, aunque en Catamergus son más frecuentes, abundantes y grandes. La presencia del género en América, y en territorios neotropicales, se reafirmó con la descripción de $I$. paranaense. Blackman \& Eastop $(2006,2011)$ no pusieron en duda la adscripción genérica de ambas especies. Los caracteres de las hembras vivíparas ápteras ahora estudiadas, tanto de I. americanum como de las nuevas especies, permiten mantener esa adscripción.

Las hembras vivíparas aladas de I. paranaense (descritas al establecerse la especie), de I. americanum (que no se habían descrito aún), de I. chiriquense y de I. holmani presentan una esclerotización dorso-abdominal limitada a las placas marginales. En contraste las aladas de las especies paleárticas de Impatientinum presentan una esclerotización dorsoabdominal más o menos extensa (Heie, 1994, pág. 79 , figs. 210 y 211).

Como mejor opción mantenemos este grupo de especies (americanum, paranaense, chiriquense, holmani y oaxacense) en el género Impatientinum. Un estudio genético-molecular de especies de ambos lados del Atlántico permitiría establecer con certeza si esa ubicación taxonómica es correcta o no lo es.

Las vivíparas ápteras de las especies americanas de Impatientinum se pueden identificar utilizando la siguiente clave dicotómica, que se presenta en inglés porque puede sustituir a partir de su disyuntiva 2 "the key to aphids on Cuphea" de Blackman \& Eastop (2006).
1. Antennae brown to dark brown ..................................... 2

- Antennae mostly pale, segment I, II, VI and apices of segment IV and V light brown to brown 3

2. Antennal segment I and II as dark as the other antennal segment. Dorsal patch brown to dark brown. Second segment of the hind tarsus $0.09 \mathrm{~mm}$ long at most (included 1.6-1.9 times on the ultimate rostral segment). Ultimate rostral segment with $15-18$ setae. Setae on the cephalic dorsum $37-55 \mu \mathrm{m}$ Impatientinum chiriquense Nieto Nafría \& Mier Durante n. sp.

- Antennal segment I and II paler than the other antennal segment. Dorsal patch pale brown. Second segment of the hind tarsus $0.10 \mathrm{~mm}$ long at least (included 1.4-1.6 times on the ultimate rostral segment). Ultimate rostral segment with 19-33 setae. Setae on the cephalic dorsum 27-37 $\mu \mathrm{m}$

Impatientinum paranaense de Carvalho et al.

3. Siphunculi with an extensive and well defined reticulation: at least 6 lines on 0.25 of total length of siphunculi. Ultimate rostral segment with 10 accessory setae at most. Setae on hind tibiae $18 \mu \mathrm{m}$ and 0.5 times the basal diameter of antennal segment III at most Impatientinum oaxacense Nieto Nafría \&

Pérez Hidalgo n. sp.

- Siphunculi with a short and not always well defined reticulation: at most 5 lines on 0.13 of total length of siphunculi. Ultimate rostral segment with 10 accessory setae at least. Setae on hind tibiae $20 \mu \mathrm{m}$ and 0.6 times the basal diameter of antennal segment III at least ....................... 4

4. Femora with a dorsal-apical portion darker than the rest of the segment and nearly as dark as tarsi and apical portion of tibiae. Body length 3.64-4.75 times the siphunculus. Ultimate rostral segment with (17)18-22 accessory setae. Flange of the siphunculus frequently straight, bad defined. First segment of tarsi with 4 or 5 setae, exceptionally 3 on some leg Impatientinum holmani Peña-Martínez \& Nieto Nafría n. sp.

- Femora pale. Body length 4.50-5.50 times the siphunculus. Ultimate rostral segment with 10-16(18) accessory setae. Flange of the siphunculus frequently large. First segment of tarsi with 3 setae, exceptionally with 4 on some leg ......... Impatientinum americanum Remaudière

\section{Agradecimientos}

Los autores agradecen al Muséum national d'Historie naturelle de París, a la Facultad de Estudios Superiores Iztacala de la Universidad Nacional Autónoma de México y a la Universidade Nacional do Paraná las facilidades para estudiar los ejemplares de sus respectivas colecciones, y también al Prof. G. Remaudière sus consejos y constante apoyo. J. M. Nieto agradece al Muséum national d'Historie naturelle de París la ayuda para su estancia de trabajo en esa institución en febrero de 2011.

Graellsia, 68(2), Diciembre 2012, pp. 313-324 - ISSN: 0367-5041 doi:10.3989/graellsia.2012.v68.070 


\section{Referencias}

Blackman, R. L. \& Eastop, V. F., 2006. Aphids on the World's herbaceous plants and shrubs. John Wiley \& Sons, Ltd. \& Natural History Museum. Chichester. 2 vols., 1440 pp.

Blackman, R. L. \& Eastop, V. F., 2011. Additions and amendments to "Aphids on the World's Plants". Zootaxa, 2774: 57-68.

de Carvalho, R. C. Z., Cardoso, J. T., Lazzari, S. M. N., 2004. A new species of Impatientinum Mordvilko (Hemiptera: Aphididae) from Brasil. Neotropical Entomology, 33(1): 39-42.

Cortés Gabaudan, F., Nieto Nafría, J. M., Favret, C., Barbagallo, S., SAno, M. \& Stekolshchikov, A. V., 2011. Etymology and gender of genus-group names. Etimología y género gramatical de los nombres de los taxones de nivel género. In: J. M. Nieto Nafría \& C. Favret (eds.). Registers of family-group and genusgroup taxa of Aphidoidea. Registros de los taxones del nivel familia y del nivel género de Aphidoidea, Hemiptera Sternorrhyncha). Universidad de León, León: 405-463.

FAVret, C. 2012, Aphid Species File. Version 1.0/4.0 [fecha de consulta: 08-05-2012]. <http://Aphid. SpeciesFile.org>

Foottit, R. G. \& RichaRds, W. R., 1993. The genera of the Aphids of Canada. Homoptera: Aphidoidea and Phylloxeroidea. The Insects and Arachnids of Canada, part 22. Centre for Land and Biological Resources Research. Ottawa. 766 pp.

HeIE, O. E., 1994, The Aphidoidea (Hemiptera) of Fennoscandia and Denmark. V. Family Aphididae: Part 2 of the tribe Macrosiphini of subfamily Aphididae. Fauna Entomologica Scandinavica, 28: 1-240.

Nieto Nafría, J. M., Favret, C., Akimoto, S.-i., Barbagallo, S., Chakrabarti, S., Mier Durante, M. P., Miller, G. L., Qiao, G.-X., Sano, M., Pérez Hidalgo, N., Stekolshchikov, A. V. \& Wegierek, P., 2011. Register of genus-group taxa of Aphidoidea. Registro de los taxones del nivel género de Aphidoidea. In: J. M. Nieto Nafría \& C. Favret (eds.). Registers of family-group and genus-group taxa of Aphidoidea. Registros de los taxones del nivel familia y del nivel género de Aphidoidea, Hemiptera Sternorrhyncha). Universidad de León, León: 81404.
Nieto Nafría, J. M. \& Mier Durante, M. P., 1998. Hemiptera, Aphididae I. Fauna Ibérica, vol. 11, Ramos, M.A. et al. (eds.). Museo Nacional de Ciencias Naturales, C.S.I.C.). Madrid. 424 pp.

Remaudière, G., 1981. Pucerons nouveaux et peu connus du Mexique. $1^{\text {re }}$ note: Latgerina orizabaensis gen. n., sp. n. et Impatientinum americanum sp. n. (Hom. Aphididae). Annales de la Societé entomologique de France, N.S., 17(4): 521-533. 